\title{
A Single-Center Randomized Controlled Trial Evaluating Timing of Preparation for Capsule Enteroscopy
}

\author{
Katherine R. Black, Wiley Truss, Cynthia I. Joiner, Shajan Peter and Frederick H. Weber Jr \\ Division of Gastroenterology and Hepatology, University of Alabama at Birmingham, Birmingham, AL, USA
}

See commentary on page 183-184

\begin{abstract}
Background/Aims: Intestinal lavage (IL) administration immediately before capsule enteroscopy (CE) is superior to lavage the day before the procedure. We aimed to determine the effect of IL timing on CE diagnostic yield.

Methods: Patients referred for CE were randomized prospectively into two equal groups according to the timing of IL with $2 \mathrm{~L}$ of polyethylene glycol solution. Group A and B underwent IL over 2 hours beginning 14 and 4 hours, respectively, before the scheduled CE. The primary outcome measure was preparation quality, measured with a predetermined validated grading scale.

Results: A total of 34 patients were randomized. The frequency of mucosal abnormalities (77\% vs. 82\%, $p=$ not significant [NS]) and diagnostic yield ( $47 \%$ vs. 53\%, $p=\mathrm{NS}$ ) were similar between the two groups. Moreover, no significant association between the quality of small bowel preparation and the timing at which the purgative for IL was administered was observed (overall fluid transparency, $p=0.936$; overall mucosal invisibility, $p=0.091$ ).
\end{abstract}

Conclusions: Day-before IL is equivalent to same-day IL in terms of overall preparation quality, proportion of complete studies to cecum, small bowel transit time, frequency of identified mucosal abnormalities, and overall diagnostic yield.

Key Words: Capsule enteroscopy; Bowel preparation

\section{INTRODUCTION}

Capsule enteroscopy (CE) was first introduced in 2000 and has become a widely applied tool for the evaluation of smallbowel pathologies. ${ }^{1}$ Given Imaging (Yoqneam, Israel) first marketed CE in 2001 and initially suggested that a clear liquid diet for 24 hours along with a 12 -hour fast prior to the procedure was sufficient preparation for CE. However, turbid intestinal fluid, residual air bubbles, food materials, and incomplete small-bowel transit of the capsule affect the diagnostic yield of

\footnotetext{
Received: March 2, 2014 Revised: June 29, 2014

Accepted: September 22, 2014

Correspondence: Frederick H. Weber Jr

Division of Gastroenterology and Hepatology, University of Alabama at Birmingham, The Kirklin Clinic, 1st Floor, Digestive Disease Center, 2000 6th Avenue South, Birmingham, AL 35233, USA

Tel: +1-205-801-8681, Fax: +1-205-801-7912, E-mail: fweber@uab.edu

(c) This is an Open Access article distributed under the terms of the Creative Commons Attribution Non-Commercial License (http://creativecommons.org/ licenses/by-nc/3.0) which permits unrestricted non-commercial use, distribution, and reproduction in any medium, provided the original work is properly cited.
}

CE. For this reason, cleaning the small intestine prior to the examination is believed to improve visibility and thus the diagnostic yield of the test. Still, no consensus on the most effective bowel preparation prior to CE exists. ${ }^{2,3}$ Studies evaluating the efficacy of various preparations and quantities used for intestinal lavage (IL) have reportedly shown that a purgative IL using polyethylene glycol (PEG) is superior to no bowel preparation, and that administration of $2 \mathrm{~L}$ of $\mathrm{PEG}$ is equivalent to $4 \mathrm{~L}^{4-6}$ However, to date no prospective studies have specifically evaluated the influence of IL timing on the quality of small bowel preparation or overall diagnostic yield.

Research on colonoscopy preparation suggests that lavage administration immediately before the procedure provides a more effective bowel preparation than that the day prior to the procedure. ${ }^{7}$ We hypothesized that since the small bowel is anatomically proximal, a bowel preparation ingested closer to the time of CE may also optimize mucosal visualization in the small bowel.

The aim of this study was to determine the optimal timing of bowel preparation prior to $\mathrm{CE}$. 


\section{MATERIALS AND METHODS}

\section{Patients and bowel preparation}

This was a prospective, randomized, single-blinded, and controlled study at a single university center. Consecutive inpatients and outpatients referred for CE within the University of Alabama at Birmingham Hospital System, between June 2011 and March 2012, were recruited for the study. Exclusion criteria were conditions that could affect intestinal motility, such as, chronic narcotic use, prokinetics use (metoclopramide, erythromycin), gastroparesis, pseudo-obstruction, and history of gastrectomy or other intestinal surgery.

Patients were prospectively randomized by the concealed envelope technique into two equal groups, according to the timing of bowel preparation with 2 L of PEG. Group A and group $\mathrm{B}$ were requested to ingest the IL over 2 hours beginning 14 hours and 4 hours before the scheduled CE, respectively. All patients were instructed to ingest a clear liquid diet the day prior to CE.

CE was performed using the Given Imaging PillCam SB wireless capsule. Each patient was prepared with sensor arrays and a data recorder, and instructed to swallow the capsule with $150 \mathrm{~mL}$ of water and $100 \mathrm{mg}$ of simethicone. CE images were recorded for the subsequent 8 hours. Patients were allowed to drink clear liquids and full liquids 2 and 4 hours after capsule ingestion, respectively.

The study protocol was approved by the Institutional Review Board (Protocol No. X110113004) at the University of Alabama at Birmingham Hospital. Written informed consent was obtained from all participants before the examination.

The primary outcome measure was small bowel preparation quality determined by a blinded single experienced reader using a predetermined quality scale adapted from Esaki et al. ${ }^{8}$ to assess both segmental and overall small bowel preparation quality. The secondary outcome measures were completion rates to the cecum, small bowel transit time (SBTT), endoscopic findings, and diagnostic yield. Lesions were categorized according to the scale of Saurin et al. ${ }^{9}$ as P0, P1, or P2 if the lesion was unrelated to bleeding, had an indeterminate relationship to bleeding, or had a high bleeding potential, respectively.

\section{Assessment of CE images}

All CE examinations were assessed by an observer (F.H.W. Jr) with experience in the interpretation of more than $500 \mathrm{CE}$ images. The digital video images, which had been downloaded to the workstation and viewed with RAPID software (Given Imaging), were analyzed to assess their quality. The reader was blinded to the patient's clinical data, including the timing of IL administration.

The grading system was derived from a previously validat- ed system. ${ }^{8}$ Accordingly, after evaluation of the gastric transit time (GTT) and SBTT on the time counter, the video images were equally divided into three segments based on the SBTT. The fluid transparency of each segment and degree of mucosal invisibility were assessed and graded by reviewing the images at low speed (10 frames/second) and at maximum speed (40 frames/second) with concurrent manual inspection of individual frames, respectively. The grade of fluid transparency and mucosal invisibility were determined according to the predominant grade in each segment and proportion of duration where air bubbles or residues disturbed visualization and interpretation by more than $50 \%$. The grading system for assessment of image quality is outlined in Table 1.

Obscure-occult bleeding was defined as iron deficiency anemia or occult blood-positive blood loss anemia, whereas obscure-overt bleeding was defined as melena or hematochezia.

Table 1. Grading System for the Assessment of Image Quality

\begin{tabular}{cl}
\hline Fluid transparency & \\
Grade 1 & Clear fluid without obscuring vision \\
Grade 2 & Slightly dark fluid minimally obscuring vision \\
Grade 3 & Opaque fluid partly obscuring vision \\
Grade 4 & Turbid fluid severely obscuring vision \\
Mucosal invisibility & \\
Grade 1 & $<5 \%$ \\
Grade 2 & $5-15 \%$ \\
Grade 3 & $15-25 \%$ \\
Grade 4 & $>25 \%$ \\
\hline
\end{tabular}

Adapted from Esaki et al., with permission from Elsevier. ${ }^{8}$

a) The percentage indicates the proportion of length of time of video image in which air bubbles or food residues disturbed more than $50 \%$ of visualization and interpretation.

Table 2. Demographic and Clinical Characteristics of Patients between Bowel Preparation Groups

\begin{tabular}{lccc}
\hline \multicolumn{1}{c}{ Variable } & $\begin{array}{c}\text { Group A } \\
\text { (14-hours before) }\end{array}$ & $\begin{array}{c}\text { Group B } \\
\text { (14-hours before) }\end{array}$ & -value \\
\hline Total patient no. & 17 & 17 & \\
Age, yr & $63 \pm 13.8$ & $56 \pm 19.7$ & 0.239 \\
Sex male & $10(59)$ & $10(59)$ & 1.000 \\
Race & & & 1.000 \\
$\quad$ White & $12(71)$ & $13(76)$ & \\
$\quad$ Black & $5(29)$ & $4(24)$ & \\
Inpatient & $7(41)$ & $14(82)$ & 0.032 \\
Indication & & & 0.035 \\
$\quad$ Obscure-occult & $9(53)$ & $2(12)$ & \\
$\quad$ Obscure-overt & $6(35)$ & $12(71)$ & \\
$\quad$ Other & $2(12)$ & $3(18)$ & \\
\hline
\end{tabular}

Values are presented as mean \pm SD or number (\%). 


\section{Statistical analysis}

In order to detect a mean difference in the grading scale of $25 \%$, with a power of $80 \%$ at a $95 \%$ significance level, a total of 34 patients (17 patients in each group) were required for enrollment. Parametric and nonparametric data were expressed as means $\pm \mathrm{SD}$ and frequencies, and groups compared using the Mann-Whitney $U$ and the chi-square tests, respectively. A $p<0.05$ was regarded as statistically significant for each test.

\section{RESULTS}

\section{Demographic data}

Demographic data are presented in Table 2. From the 49 patients that were evaluated for inclusion in the study, 15 were excluded, and from those, 11 were excluded based on the presence of one of the predetermined exclusion criteria, whereas four chose not to participate despite meeting the inclusion criteria. Thus, a total of 34 patients were enrolled and randomized to either group A $(n=17)$ or group B $(n=17)$, to receive the bowel preparation 14 or 4 hours before $\mathrm{CE}$, respectively, during the study period. The patients mean age was 59.6 years (range, 21 to 80); 26.5\% were African American and 73.5\% Caucasian; $41.2 \%$ were women and $58.8 \%$ men; $61.8 \%$ were inpatients and $38.2 \%$ outpatients; $55.9 \%$ underwent CE for GI bleeding and $44.1 \%$ underwent $\mathrm{CE}$ for an indication other than GI bleeding. No significant differences between the two groups were found with respect to age, race, or gender. Despite random allocation, 14 patients (82\%) in group B were inpatients versus seven $(41 \%)$ in group A ( $p=0.032)$. Nine patients (53\%) in group $\mathrm{A}$ underwent $\mathrm{CE}$ for obscure-occult bleeding versus two (12\%) in group B, whereas six patients (35\%) in group $\mathrm{A}$ underwent $\mathrm{CE}$ for obscure-overt bleeding versus 12 $(71 \%)$ in group $\mathrm{B}(p=0.035)$.

\section{Image quality}

Image quality declined steadily in both groups as the capsule advanced towards the terminal ileum. As illustrated in Figs. 1, 2, mean segmental and overall grades for fluid transparency and mucosal visibility were not statistically different between the two groups.

Regardless of the timing of the bowel preparation, when comparing the overall quality of bowel preparation for all patients, there was no statistically significant association with the indication for $\mathrm{CE}$ (bleeding vs. non-bleeding) or with inpatient versus outpatient status. However when looking specifically at mucosal invisibility in segment 1 and segment 2 , inpatients did have a worse bowel preparation compared to those that were given their bowel preparation and completed CE as an outpatient ( $p=0.05$ and $p=0.018$, respectively).

\section{CE findings}

CE findings are summarized in Table 3. Complete and incomplete small bowel visualization at $\mathrm{CE}$ completion was ob-

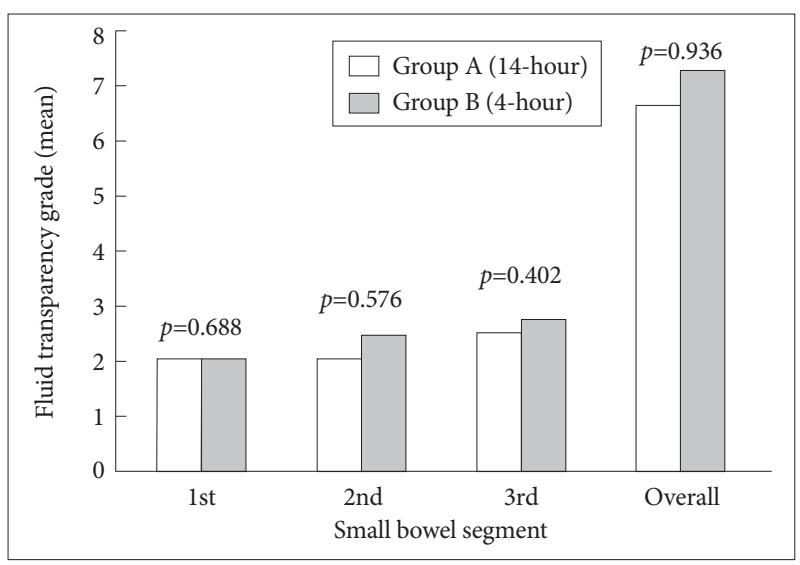

Fig. 1. Fluid transparency assessment by segment and overall grade.

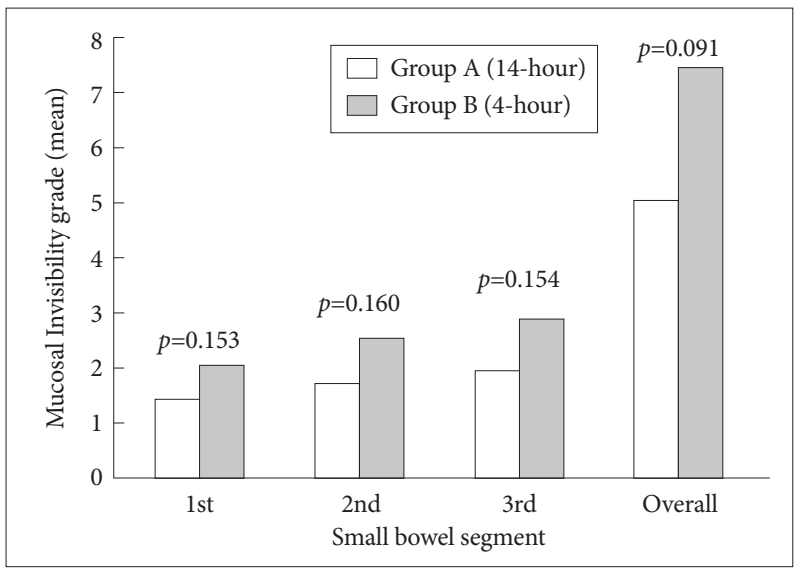

Fig. 2. Mucosal invisibility assessment by segment and overall grade.

Table 3. Capsule Endoscopy Findings of Patients between Bowel Preparation Groups

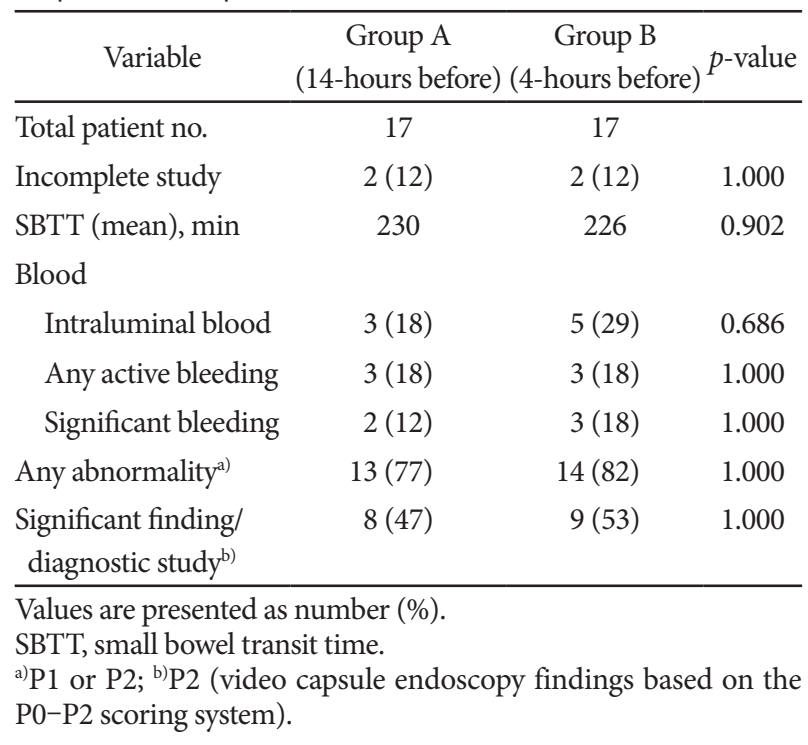


tained in 15 patients (88\%) and two patients, respectively, in each group. Mean SBTT was 230 minutes in group A and 226 minutes in group B ( $p=0.902)$. Mucosal abnormalities (Saurin $\mathrm{P} 1$ or $\mathrm{P} 2$ lesions $^{9}$ ) were found in 13 patients (77\%) and 14 $(82 \%)$ in group A and group B, respectively $(p=1.0)$. The diagnostic yield, defined as a clinically relevant abnormality (Saurin P2 lesions), was similar in both groups (47\% vs. $53 \%$ ). No differences with respect to findings of any intraluminal blood, active bleeding, or bleeding significant enough to obscure capsule luminal view and preparation quality score, were observed between groups.

\section{DISCUSSION}

CE is a non-invasive and convenient method used in the examination of the small intestinal mucosa. However, its diagnostic utility is limited by intestinal fluid turbidity, residual air bubbles, food material, and incomplete small bowel capsular transit limited by the temporal capacity of a power source. Thus, optimizing visualization to ensure satisfactory luminal views and maximize the diagnostic yield is imperative.

Several meta-analyses have evaluated studies assessing various IL modalities. Niv at al. ${ }^{10}$ included eight studies comparing sodium phosphate, PEG, or simethicone with no preparation, and demonstrated that the quality of small bowel visualization was improved in the groups using an IL. Similarly, Rokkas et al. ${ }^{11}$ evaluated 12 studies comparing PEG or simethicone versus no preparation and found that the administration of preparations improved small bowel visualization and diagnostic yield. No differences regarding video CE GTT and video CE SBTT were observed between groups.

Previous studies have suggested that the PEG purgative is effective for $\mathrm{CE}^{4-6,12-14}$ and that $2 \mathrm{~L}$ PEG is equivalent to $4 \mathrm{~L}^{15,16}$ when given the day prior to the exam. Comparisons of PEG with sodium phosphate have yielded conflicting results, ${ }^{11}$ while simethicone given alone or with purgatives seems to improve at least proximal small bowel visualization. ${ }^{17-21}$ Prokinetics such as metoclopramide, erythromycin, bisacodyl, lubiprostone, and mosapride have failed to improve CE completion rates. ${ }^{22-26}$ However, the use of chewing gum/sham feeding, a real-time viewer, and temporary reduction of image capture rate while in the stomach, have been suggested as techniques that improve CE completion rates. ${ }^{27-29}$

Purgative administration timing may be a critical factor for visualization and $\mathrm{CE}$ completion rates. Most purgative studies have administered them the day prior to the CE study. Yet, consensus on colonoscopy preparation suggests that, compared with lavage administration the day before $\mathrm{CE}$, administering the lavage several hours before commencing the procedure clearly enhanced colonic preparation quality. Since the small intestine is anatomically proximal, we hypothesized that a similar lavage timing concept would be advantageous for CE small intestinal visualization and perhaps transit. Our study is the first to assess the most optimal IL timing for CE. Although a previous study by Ito et al. reported IL administration after capsule ingestion, ${ }^{30}$ the optimal IL timing was not addressed because the comparison group received no IL. However, our study demonstrates that same-day IL is not advantageous for CE small intestinal visualization, transit, or completion rate. Moreover, the diagnostic yield did not improve regardless of whether Saurin P1 or P2 lesion categories were considered. Although not statistically significant, a steady degradation of visualization quality was observed in both groups, as the capsule advanced toward the terminal ileum. This suggests that with regard to luminal visualization, proximal bilious contents are less problematic than more distal intestinal chyme, and that sameday lavage does not seem to improve this issue. Thus, techniques to reduce this distal degradation of preparation quality are needed.

Our demonstration that a rapid IL followed by same-day CE is equivalent to day-before IL offers the opportunity for a more expeditious performance of CE in the setting of acute GI bleeding. This may reduce the length of hospital stay in selected patients, and therefore, overall costs for obscure GI bleeding management.

As mentioned above, Ito et al. ${ }^{30}$ evaluated PEG lavage timing further in a prospective controlled study in which PEG lavage was administered 2 hours after capsule ingestion, with superior CE visualization quality observed in each small intestinal segment when compared to a group taking no lavage/ purgative. Further trials comparing IL administration before and several hours after capsule ingestion are needed.

Our study has several potential limitations. First, 61.8\% (21 of 34 patients) were inpatients, many of whom had recent overt gastrointestinal (GI) bleeding, with intraluminal blood potentially being a confounder in our preparation quality analysis. However, we believe the effect of intraluminal blood on preparation quality analysis was minimal, as there was no significant difference between groups with regard to luminal blood quantities, significant enough to obscure views. Secondly, randomization allocated more inpatients to group $B$ than to group $\mathrm{A}$, and this could be a confounder with respect to the effects of comorbidities on capsule visualization. Thirdly, no universally accepted and validated grading scale for CE visualization quality assessment currently exists. The grading scale used in this analysis showed excellent interobserver agreement as previously evaluated by Esaki et al. ${ }^{8}$ Nonetheless, the assessment of fluid transparency has a subjective component, perhaps more significant than that of the mucosal invisibility assessment. However, we used the same fluid transparency 
criteria for both groups. Further studies are needed to determine a simple grading system with limited intraobserver and interobserver variability.

We conclude that the difference in the quality bowel preparation between day-before PEG and same-day IL before CE is not significant. Furthermore, IL timing has no effect on completion rates to the cecum, SBTT, frequency of identified mucosal abnormalities, or diagnostic yield. The timing to schedule a CE may be decreased by allowing the patient to ingest a lavage preparation closer to the time of examination. This strategy may prove useful in cases of emergent CE and may reduce the length of hospital stay in selected patients with obscure GI bleeding. Further trials are necessary to determine the optimal timing and technique for CE lavage.

\section{Conflicts of Interest}

The authors have no financial conflicts of interest.

\section{REFERENCES}

1. Iddan G, Meron G, Glukhovsky A, Swain P. Wireless capsule endoscopy. Nature 2000;405:417.

2. de Franchis R, Avgerinos A, Barkin J, Cave D, Filoche B; ICCE. ICCE consensus for bowel preparation and prokinetics. Endoscopy 2005;37: 1040-1045.

3. Mergener K, Ponchon T, Gralnek I, et al. Literature review and recommendations for clinical application of small-bowel capsule endoscopy, based on a panel discussion by international experts. Consensus statements for small-bowel capsule endoscopy, 2006/2007. Endoscopy 2007; 39:895-909.

4. Dai N, Gubler C, Hengstler P, Meyenberger C, Bauerfeind P. Improved capsule endoscopy after bowel preparation. Gastrointest Endosc 2005; 61:28-31.

5. Viazis N, Sgouros S, Papaxoinis K, et al. Bowel preparation increases the diagnostic yield of capsule endoscopy: a prospective, randomized, controlled study. Gastrointest Endosc 2004;60:534-538.

6. Kantianis A, Karagiannis S, Liatsos C, et al. Comparison of two schemes of small bowel preparation for capsule endoscopy with polyethylene glycol: a prospective, randomized single-blind study. Eur J Gastroenterol Hepatol 2009;21:1140-1144.

7. Kilgore TW, Abdinoor AA, Szary NM, et al. Bowel preparation with split-dose polyethylene glycol before colonoscopy: a meta-analysis of randomized controlled trials. Gastrointest Endosc 2011;73:1240-1245.

8. Esaki M, Matsumoto T, Kudo T, Yanaru-Fujisawa R, Nakamura S, Iida M. Bowel preparations for capsule endoscopy: a comparison between simethicone and magnesium citrate. Gastrointest Endosc 2009;69:94-101.

9. Saurin JC, Delvaux M, Gaudin JL, et al. Diagnostic value of endoscopic capsule in patients with obscure digestive bleeding: blinded comparison with video push-enteroscopy. Endoscopy 2003;35:576-584.

10. Niv Y, Niv G, Wiser K, Demarco DC. Capsule endoscopy: comparison of two strategies of bowel preparation. Aliment Pharmacol Ther 2005;22: 957-962.

11. Rokkas T, Papaxoinis K, Triantafyllou K, Pistiolas D, Ladas SD. Does purgative preparation influence the diagnostic yield of small bowel video capsule endoscopy? A meta-analysis. Am J Gastroenterol 2009;104:
219-227.

12. van Tuyl SA, den Ouden H, Stolk MF, Kuipers EJ. Optimal preparation for video capsule endoscopy: a prospective, randomized, single-blind study. Endoscopy 2007;39:1037-1040.

13. Belsey J, Crosta C, Epstein O, et al. Meta-analysis: efficacy of small bowel preparation for small bowel video capsule endoscopy. Curr Med Res Opin 2012;28:1883-1890.

14. Koulaouzidis A, Rondonotti E, Karargyris A. Small-bowel capsule endoscopy: a ten-point contemporary review. World J Gastroenterol 2013; 19:3726-3746.

15. Koornstra JJ. Bowel preparation before small bowel capsule endoscopy: what is the optimal approach? Eur J Gastroenterol Hepatol 2009;21: 1107-1109.

16. Song HJ, Moon JS, Do JH, et al. Guidelines for bowel preparation before video capsule endoscopy. Clin Endosc 2013;46:147-154.

17. Rey JF, Repici A, Kuznetsov K, Boyko V, Aabakken L. Optimal preparation for small bowel examinations with video capsule endoscopy. Dig Liver Dis 2009; 41:486-493.

18. Fang YH, Chen CX, Zhang BL. Effect of small bowel preparation with simethicone on capsule endoscopy. J Zhejiang Univ Sci B 2009;10:46-51.

19. Shiotani A, Opekun AR, Graham DY. Visualization of the small intestine using capsule endoscopy in healthy subjects. Dig Dis Sci 2007;52: 1019-1025.

20. Wei W, Ge ZZ, Lu H, Gao YJ, Hu YB, Xiao SD. Purgative bowel cleansing combined with simethicone improves capsule endoscopy imaging. Am J Gastroenterol 2008;103:77-82.

21. Postgate A, Tekkis P, Patterson N, Fitzpatrick A, Bassett P, Fraser C. Are bowel purgatives and prokinetics useful for small-bowel capsule endoscopy? A prospective randomized controlled study. Gastrointest Endosc 2009;69:1120-1128.

22. Almeida N, Figueiredo P, Freire P, et al. The effect of metoclopramide in capsule enteroscopy. Dig Dis Sci 2010;55:153-157.

23. Caddy GR, Moran L, Chong AK, Miller AM, Taylor AC, Desmond PV. The effect of erythromycin on video capsule endoscopy intestinaltransit time. Gastrointest Endosc 2006;63:262-266.

24. Franke A, Hummel F, Knebel P, et al. Prospective evaluation of small bowel preparation with bisacodyl and sodium phosphate for capsule endoscopy. World J Gastroenterol 2008;14:2061-2064.

25. Hooks SB 3rd, Rutland TJ, Di Palma JA. Lubiprostone neither decreases gastric and small-bowel transit time nor improves visualization of small bowel for capsule endoscopy: a double-blind, placebo-controlled study. Gastrointest Endosc 2009;70:942-946.

26. Wei W, Ge ZZ, Lu H, Gao YJ, Hu YB, Xiao SD. Effect of mosapride on gastrointestinal transit time and diagnostic yield of capsule endoscopy. J Gastroenterol Hepatol 2007;22:1605-1608.

27. Apostolopoulos P, Kalantzis C, Gralnek IM, Liatsos C, Tsironis C, Kalantzis N. Clinical trial: effectiveness of chewing-gum in accelerating capsule endoscopy transit time: a prospective randomized, controlled pilot study. Aliment Pharmacol Ther 2008;28:405-411.

28. Lai LH, Wong GL, Lau JY, Sung JJ, Leung WK. Initial experience of real-time capsule endoscopy in monitoring progress of the videocapsule through the upper GI tract. Gastrointest Endosc 2007;66:1211-1214.

29. Liao Z, Li ZS, Xu C. Reduction of capture rate in the stomach increases the complete examination rate of capsule endoscopy: a prospective randomized controlled trial. Gastrointest Endosc 2009;69(3 Pt 1):418-425.

30. Ito T, Ohata K, Ono A, et al. Prospective controlled study on the effects of polyethylene glycol in capsule endoscopy. World J Gastroenterol 2012;18:1789-1792. 